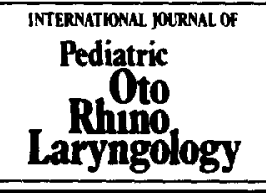

International Journal of Pediatric Otorhinolaryngology

28 (1993) 51-61

\title{
Radiation therapy as an alternative to surgery in the management of intracranial juvenile nasopharyngeal angiofibroma ${ }^{\dagger}$
}

\author{
Brian J. Wiatrak*a ${ }^{*}$ Charles F. Koopmann ${ }^{\mathrm{a}}$, Andrew T. Turrisi ${ }^{\mathrm{b}}$ \\ "Department of Otolaryngology-Head and Neck Surgery, hepartment of Radiation Oncology. \\ University of Michigan Medical Center Ann, Arbor, MI, USA
}

(Received 27 July 1992; revision received 4 January 1993; accepted 10 January 1993)

\begin{abstract}
Juvenile nasopharyngeal angiofibroma is a benign, vascular tumor which typically presents in adolescent males. Although surgical resection is usually recommended for the management of this tumor, external beam radiation therapy has also been advocated in the literature. We report three cases of large juvenile nasopharyngeal angiofibromas with extensive intracranial extension primarily managed with external beam radiation therapy. Although there was nut complete resolution of the tumors, there was significant alleviation of symptomatology with no serious side effects from the radiation therapy. Based on these cases, we feel that external beam radiation therapy in the management of extensive juvenile nasopharyngeal angiofibromas with intracranial extension is warranted in certain select cases.
\end{abstract}

Key words: Juvenile nasopharyngeal angiofibroma; Intracranial extension; Radiation therapy

\section{Introduction}

Juvenile nasopharyngeal angiofibroma (JNA) is a benign, highly vascularized tumor with an aggressive growth pattern. It accounts for $0.5 \%$ of all head and neck tumors [2] and primarily affects adolescent males. Both spontaneous involution with

\footnotetext{
* Corresponding author, 2451 Taubman Center, Box 0313, 1500 East Medical Center Drive, Ann Arbor, MI 48109-0313, USA.

${ }^{\dagger}$ This paper was presented at the Society for Ear, Nose, and Throat Advances in Children, Galveston, Texas in December, 1991.
} 
regression and malignant transformation have been reported by Spector [18]. Most otolaryngologist-head and neck surgeons advocate the surgical extirpation of these tumors, however, radiation therapy is advocated in certain select instances. The literature abounds with scientific papers which strongly advocate both treatment modalities. It appears that both surgical resection and radiation therapy have definite roles in specific clinical situations. We report three cases of juvenile nasopharyngeal angiofibroma with extensive intracranial involvement. All three cases were treated with megavoltage radiation therapy and have not necessitated surgical intervention other than for tissue biopsy.

\section{Case report 1}

A 15-year-old white male presented with progressive symptoms of right nasal obstruction, intermittent epistaxis, frontal headache, and right hearing loss.

Examination was remarkable for a large, completely obstructing right nasal mass (Fig. 1) associated with right facial edema and a right middle ear effusion. Cranial nerves II through XII were grossly intact. There was no evidence of proptosis or other orbital abnormalities. Mirror examination of the nasopharynx revealed a large nasopharyngeal mass, primarily on the right side. The remainder of the examination was unremarkable.

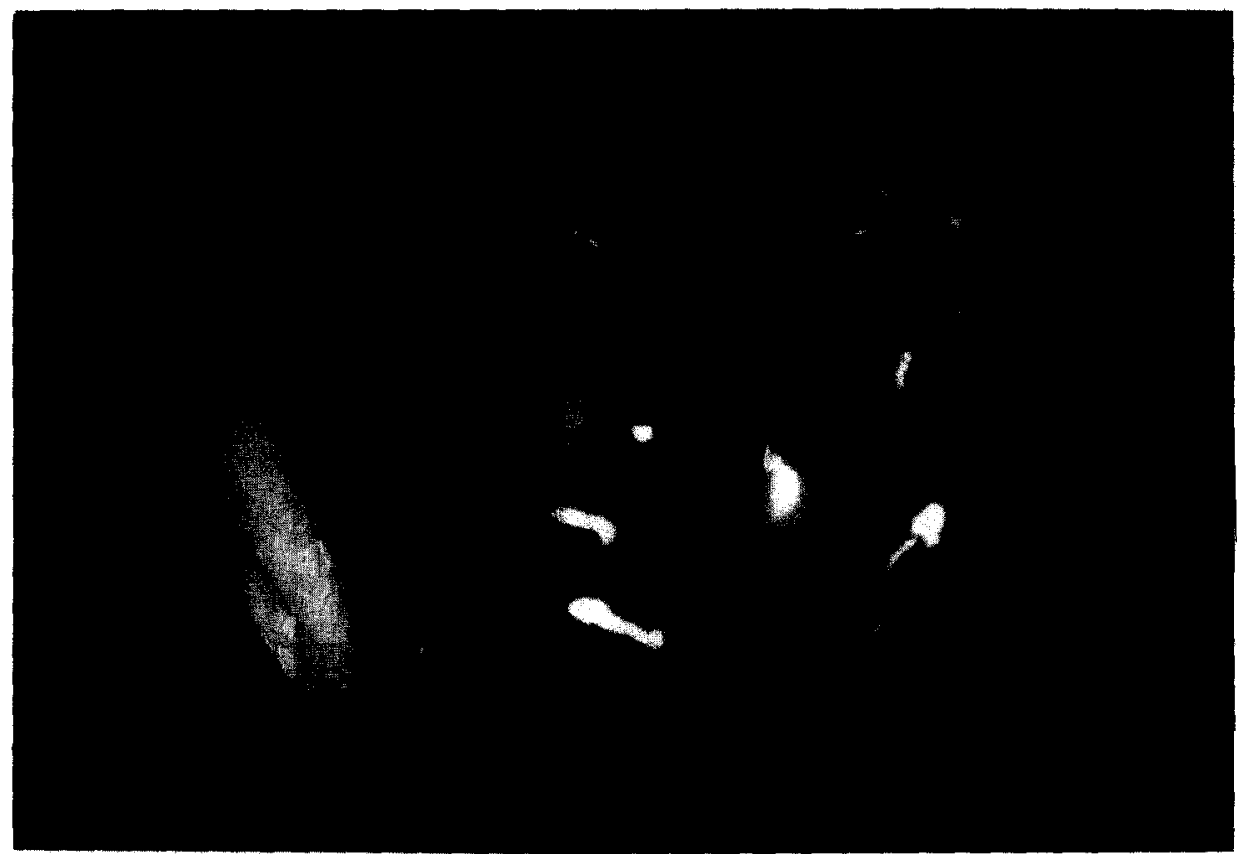

Fig. 1. Left completely obstructing haemorrhagic right nasal mass from Case 1 pushing the septum (arrow) towards the right side. 
A CT scan with contrast of the paranasal sinuses was obtained (Fig. 2), revealing a large mass involving the right nasal cavity, nasopharynx, extending into the infratemporal fossa and into the region of the inferior orbital fissure. There was superior extension through the superior orbital fissure into the region of the middle cranial fossa, involving the lateral aspect of the cavernous sinus. The significant vascularity of the lesion was highly suggestive of juvenile nasopharyngeal angiofibroma.

Carotid angiography was obtained, revealing the mass to have significant vascularization from both the internal and external carotid systems bilaterally. Embolization was performed at this time. An ophthalmologic evaluation revealed a normal neuro-ophthalmologic examination.

A biopsy was performed in the operating room, however, the final results of the biopsy revealed necrosis and inflammation with no obvious tumor.

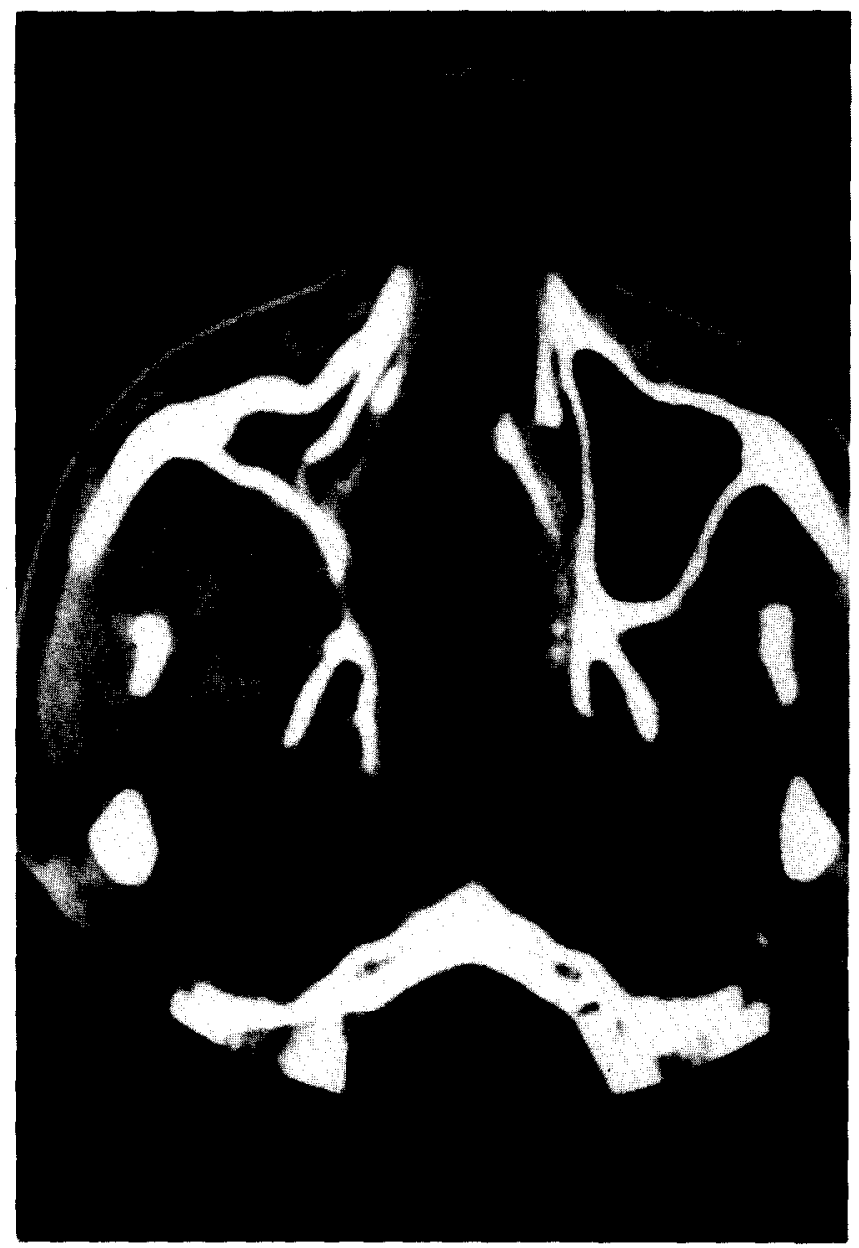

Fig. 2a. Axial section of a computed tomography scan from Case 1 revealing large vascular right nasal and nasopharyngeal mass with involvement of the pterygomaxillary space. 


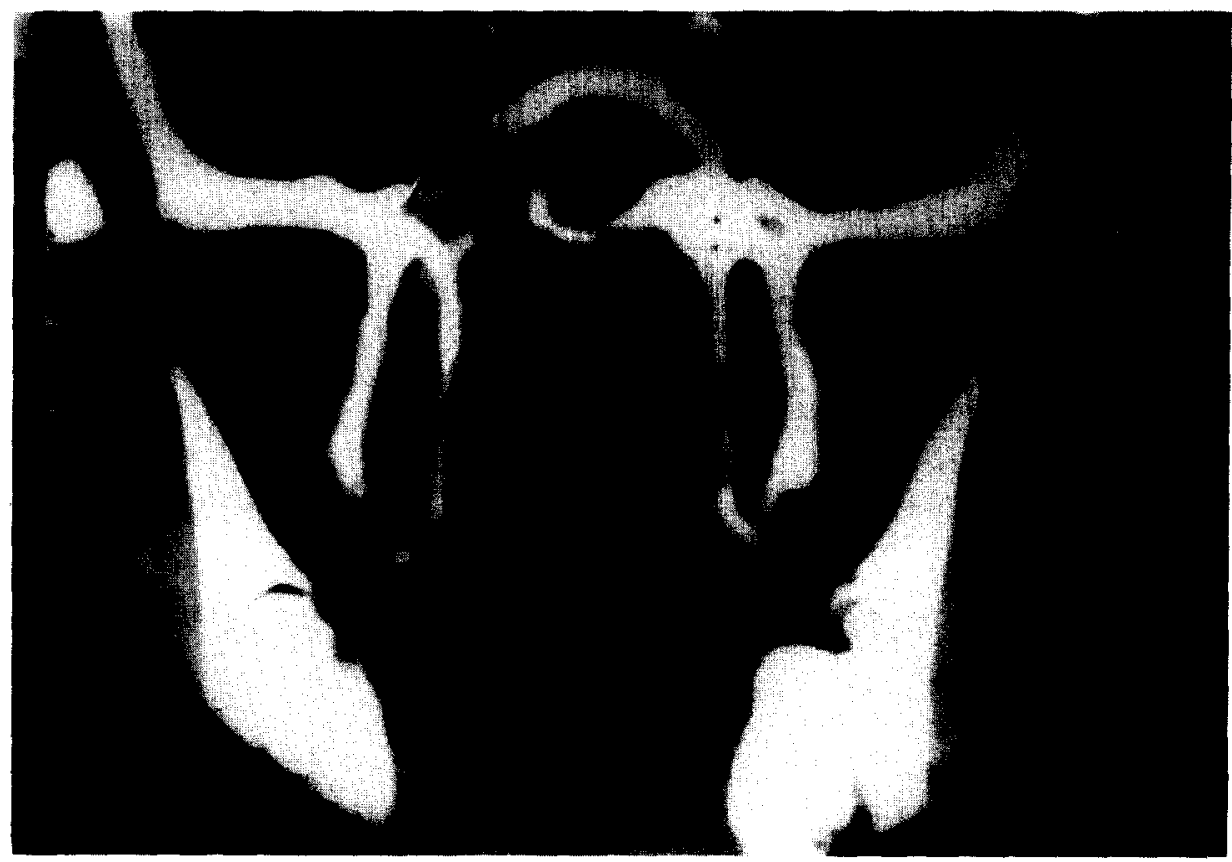

Fig. 2b. Coronal section of the same patient, revealing tumor involvement in the region of the right cavernous sinus (arrow).

Neurosurgical consultation was obtained. Based on the extent of the lesion with its intracranial involvement around the intracranial carotid artery and cavernous sinus, it was elected to pursue radiation therapy. The patient received $4000 \mathrm{cGy}$ of megavoltage radiation therapy over a 4-week period. Following therapy, a CT scan was obtained, revealing a significant decrease in the size of the tumor mass (Fig. 3). Nasal examination revealed a small mucosally covered nasal mass with minimal obstruction on the right side. The nasopharyngeal mass, although still present, was much smaller on flexible nasopharyngoscopy. Twenty months following radiation therapy, the patient has very mild right nasal obstruction, no epistaxis, resolution of facial swelling and headaches. A recent CT scan demonstrated a further decrease in the mass size.

\section{Case report 2}

A 13-year-old white male was treated for progressive nasal obstruction over 6 months and diagnosed with inhalant allergy. His history was also significant for recurrent epistaxis, frontal headaches, and numerous episodes of sinusitis treated with antibiotics. A plain sinus radiograph was obtained, revealing a nasopharyngeal mass and right maxillary sinus opacification. A biopsy by a referring otolaryngologist was consistent with JNA. The patient was referred to the Otolaryngology Service at this 


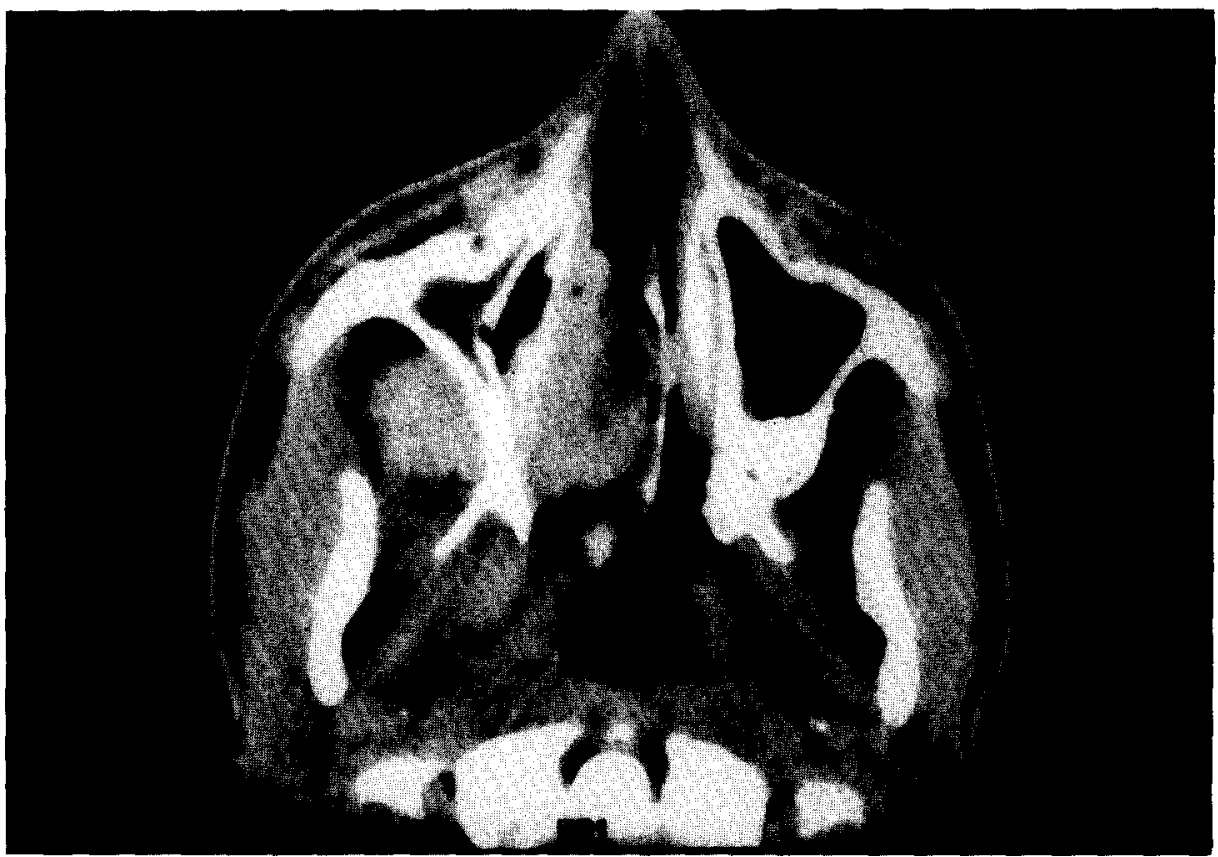

Fig. 3. Coronal computed tomography scan from Case 18 months following radiation therapy.

time. A large mass was noted within the nasopharynx and the right nasal cavity. A CT scan (Fig. 4) was obtained, revealing a large enhancing mass involving the right nasal cavity and nasopharynx, with involvement of the pterygomaxillary space, infratemporal fossa, and invasion of the inferior orbital fissure with encroachment on the optic foramen. Erosion of the lesser wing of the sphenoid bone was also noted. The MRI demonstrated significant intracranial involvement in the region of the right cavernous sinus.

Due to the extensive intracranial involvement, the patient was treated with 5040 cGy megavoltage radiation therapy over a 5-week period. A follow up CT scan revealed significant decrease in the size of the mass (Fig. 5). His headaches and epistaxis completely resolved. The mass, although persistent in the nasopharynx, was much smaller post-radiation therapy. During radiation therapy, the patient developed mild glaucoma of the right eye, which eventually resolved. A neuroophthalmologic evaluation was significant for mild right optic atrophy. It is not clear whether this was secondary to radiation therapy or the extensive orbital involvement of the tumor. The patient currently has no recurrence of his symptoms 2.5 years following radiation therapy and his last MRI scan was unchanged.

\section{Case report 3}

A 10-year-old white male presented with a 5-year history of frontal headaches which have been treated as childhood migraine. He had a history of chronic mouth 


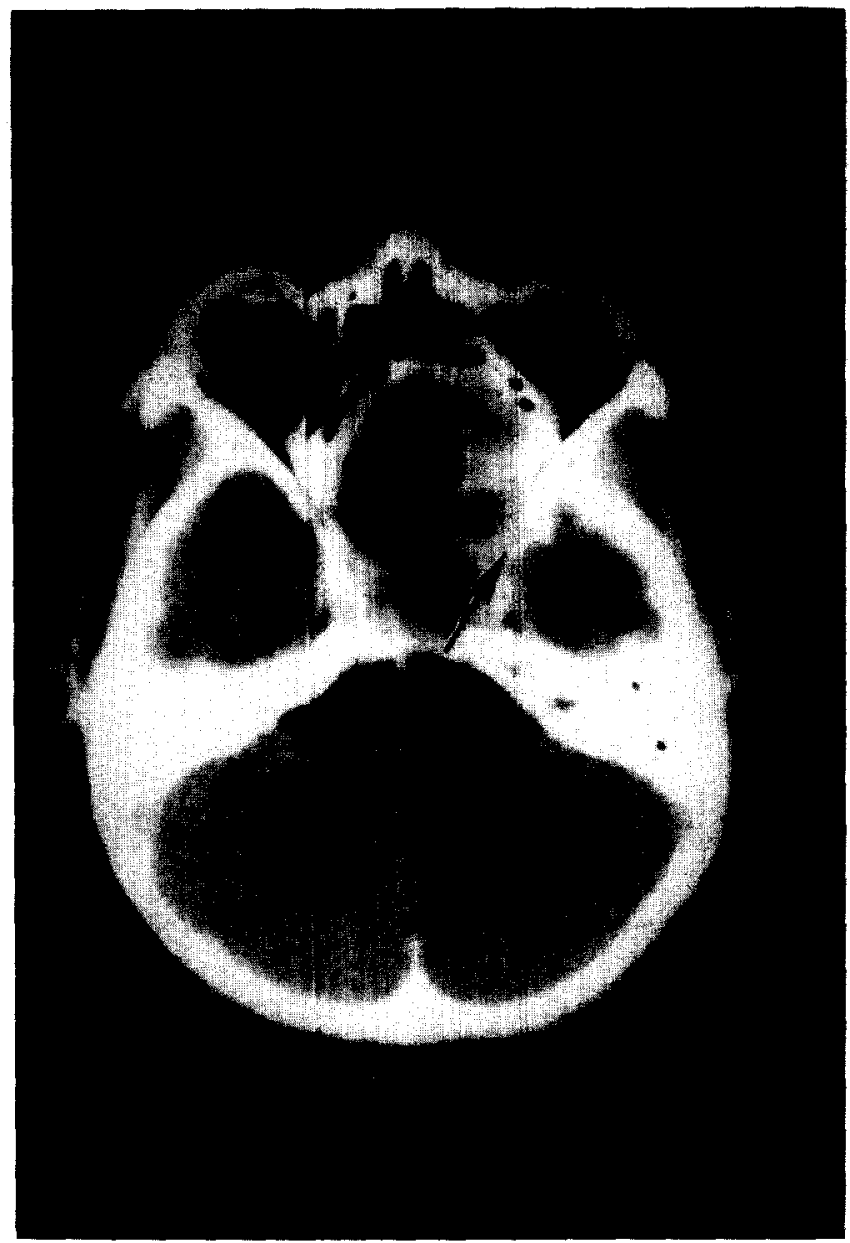

Fig. 4. Axial CT scan from Case 2. Note the involvement in the region of the left cavernous sinus (arrow).

breathing over the previous year and had been placed on nasal steroids, nasal decongestants, and immunotherapy after allergy testing had been performed. He presented acutely with a 10-day history of progressive left proptosis, blurred vision, and left orbital cellulitis. Examination at this time revealed a left Marcus Gunn pupil and right papilledema which was consistent with Foster-Kennedy syndrome. Neurosurgery was immediately consulted and an emergent CT scan with contrast was obtained, revealing a large enhancing mass which involved the nasopharynx, left nasal cavity, sphenoid sinus, and floor of the sella turcica. There was intracranial extension of the mass into the region of the cavernous sinus. Other than the left orbital findings, there were no other cranial nerve abnormalities. Intravenous corticosteroids were administered. An ophthalmologic evaluation revealed a large central scotoma of the left field of vision. Otolaryngology was consulted at this time and examination under anesthesia was performed. A large, mucosally covered, 


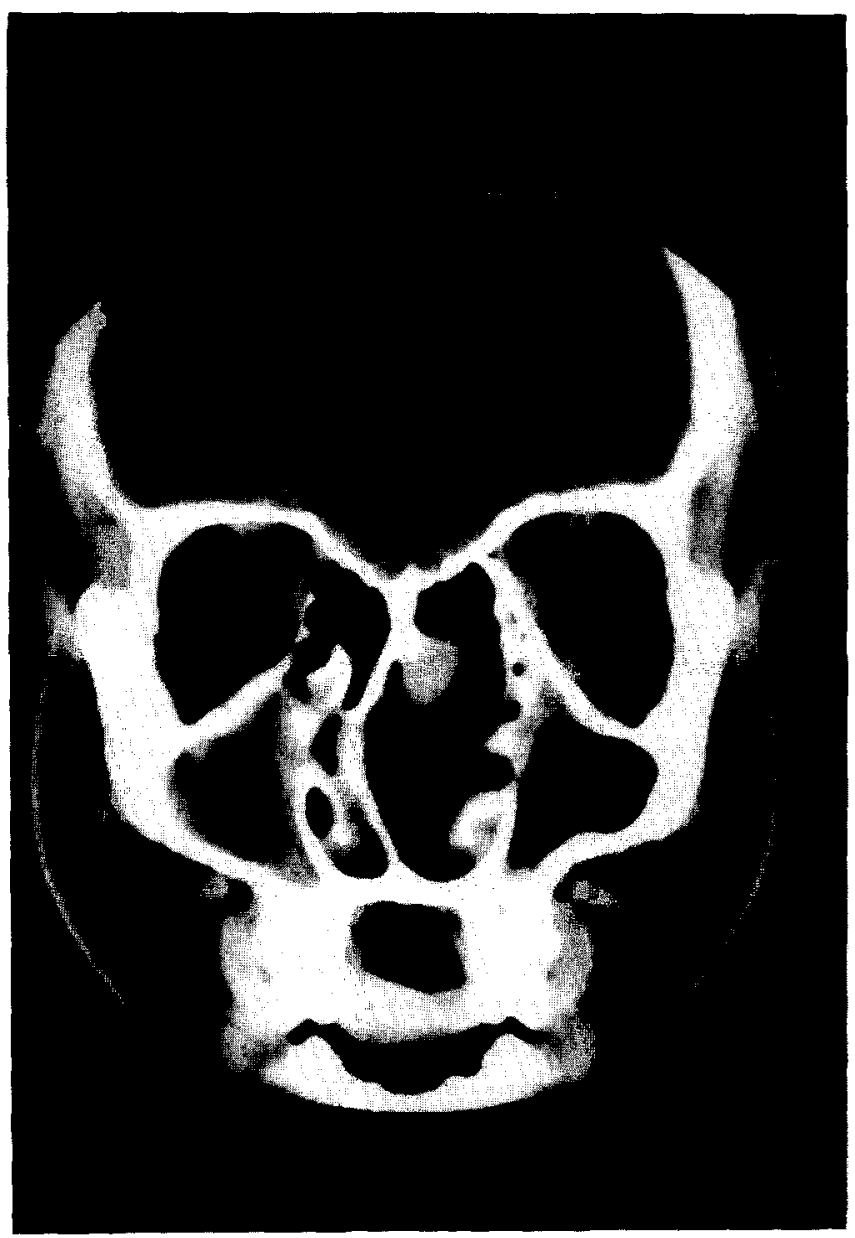

Fig. 5. Coronal section computed tomography scan 4.5 years following radiation therapy demonstrating a significant decrease in the size of the tumor mass.

nasopharyngeal mass was noted, which on needle aspiration, demonstrated significant vascularity. A biopsy was not performed at this time due to the extensive bleeding from the needle aspirate. Carotid angiography was performed, revealing a large vascular mass centered in the region of the left pterygopalatine fossa and posterior nasal cavity on the left side. Intracranial extension to the cavernous sinus and sella turcica was noted. There was significant vascular supply from both internal and external carotid systems bilaterally.

The patient began radiation therapy with a total dose of $3660 \mathrm{cGy}$ over 4 weeks and was gradually tapered off his steroids. During radiation therapy, his symptoms of nasal obstruction, headache, and proptosis decreased significantly, with no episodes of epistaxis. A follow-up CT scan revealed a persistent mass which was significantly decreased in size. Five years following radiation therapy, a small left nasal polypoid mass persists with no significant nasal obstruction. Visual acuity pro- 
blems remain in the left eye, however, there have been no complications secondary to the radiation therapy. An MRI scan performed 4.5 years following radiation therapy was unchanged from his previous examinations.

\section{Discussion}

The literature is divided over the optimal management for juvenile nasopharyngeal angiofibroma. Although most investigators feel that surgical extirpation is the treatment modality of choice, a strong argument has been made for definitive treatment utilizing megavoltage radiation therapy $[3,4,9,10,14,17]$. The controversy centers on the potential operative mortality and postoperative morbidity versus the potential complications and long-term risks of radiation to the adolescent head and neck. Curative rates of approximately $80 \%$ have been reported for both treatment modalities $[8,10,11]$. Our three cases demonstrate that radiation therapy is a reasonable alternative to surgery in cases of extensive tumors with intracranial extension involving the region of the cavernous sinus. Although marked regression of the tumor may result from this technique, persistent tumor is a common finding following radiation therapy, and may remain indefinitely after therapy.

The tumor begins its growth at the posterior superior lateral margin of the nasal cavity and eventually grows to fill the posterior nasal cavity completely and then extends to fill the nasopharynx. Lateral extension then occurs through the sphenopalatine foramen into the pterygomaxillary fossa and subsequently into the infratemporal fossa. Superior extension into the inferior orbital fissure may occur, leading to proptosis and potential encroachment on the optic nerve in the region of the optic foramen. Once the tumor has entered the orbit, it may extend into the middle cranial fossa through the superior orbital fissure, and extend lateral to the cavernous sinus. Often at this point, destruction of the greater wing of the sphenoid bone will be noted on CT scan. The tumor usually remains extradural, although invasion of the dura may occur. Another potential route of spread into the cranial fossa is through the roof of the sphenoid sinus. Superior extension into the sphenoid sinus may lead to erosion of the roof of the sphenoid sinus extending into the region of the pituitary gland and the sella turcica lying medial to the cavernous sinus. Blindness may occur from tumor growth in this area due to pressure on the optic chiasm.

Although spontaneous regression of angiofibroma has been mentioned in the literature, Neel et al. [15] in a review of 120 cases reported no evidence of spontaneous regression.

The blood supply is derived from the external carotid arterial system, most commonly the internal maxillary artery. As the tumor enlarges, bilateral vascularization occurs and vascular arterial feeders from the internal carotid system may become involved. Massive tumors most likely will have vascularization from both the internal and external carotid systems bilaterally.

The typical clinical presentation of JNA is an adolescent male with a progressive history of unilateral nasal obstruction and epistaxis which can become severe at times. Other findings may be facial swelling, proptosis, headaches, hearing loss secondary to a middle ear effusion, and facial pain. Any adolescent male with persistent 
nasal obstruction not responsive to medical therapy should be evaluated thoroughly for the cause of nasal obstruction, including a thorough examination of the nasopharynx, either by mirror or flexible nasopharyngoscopy.

The diagnostic work-up after a thorough head and neck examination in cases of suspected JNA should consist of computed tomography with contrast. JNA has a classic appearance on CT scan, with a large diffusely enhancing mass which may show evidence of bony erosion in the region of the pterygoid plates, sphenopalatine foramen, and superior aspect of the sphenoid sinus tumors. Spector states that MRI is superior to CT scan in assessing CNS invasion of angiofibroma. In addition, MRI may better delineate soft tissue extent of the tumor and will also have a classic appearance on enhanced scans. Angiography should be performed in all cases of suspected JNA. Angiography will delineate with detail the vascular supply of the tumor and assess the extent, if any, of intracranial extension. In addition, embolization of the tumor, most commonly with Gelfoam, may significantly decrease the vascularity of the tumor preoperatively if done within $48 \mathrm{~h}$ of surgery. The role of embolization pre-radiation therapy is not clear at this time. The necrotic appearance of the biopsy specimen in Case 1 is probably secondary to the prior embolization.

The role of biopsy in the management of JNA is controversial. In the past, it was felt that biopsy was not necessary since JNA has a classic appearance based on its clinical findings, CT and MRI appearance, and angiography. Burkey et al. [5] reported a nasopharyngeal mass in a 6-year-old boy with findings suggestive of JNA based on CT, MRI, and angiography. Transantral biopsy revealed embryonal rhabdomyosarcoma. We feel that tissue biopsy should be obtained if possible in all cases of JNA which are to undergo radiation therapy to rule out potentially malignant lesions. If surgical resection is planned, biopsy is not necessary. Consensus has yet to be reached on this issue.

Numerous surgical approaches exist for the management of JNA $[1,6,7,11,12$, $15,18,19]$. Most common are the lateral rhinotomy, transpalatal and extended transantral approach, and midface degloving. Less commonly used techniques include the infratemporal fossa approach and the transmandibular approach to the nasopharynx [13]. In cases of intracranial extension, a combined craniotomy with the above mentioned procedures will be necessary. Most large surgical series of JNA report recurrence rates of $20 \%$. Although the mortality from resection of JNA is relatively low, intraoperative bleeding can be a significant problem, even under the best of circumstances, necessitating multiple blood transfusions intraoperatively. Intraoperative deaths have been reported for resection of JNA $[16,20]$.

In 1970, Briant et al. [4] first published results from Princess Margaret Hospital in Toronto of 28 cases of JNA treated by radiation therapy. Treatment consisted of 3000-3500 cGy of megavoltage radiation therapy for 3 weeks. The experience was again updated in 1984 by Cummings et al. [10] reporting 55 patients. Eighty percent of these patients had permanent tumor control following a single course of radiation therapy. In the 11 patients with regrowth of tumor, three patients underwent surgical resection, and eight received a second course of radiation therapy, with tumor control in both groups. The length of follow-up in this series ranged from 3 to 26 years.

McGahan et al. [14] reported 15 patients with JNA treated with radiation therapy at Baylor College of Medicine from 1973 to 1986. Ten patients were treated with pri- 
mary radiation therapy, and five received postoperative radiation therapy for recurrence of tumor. All ten patients who were referred for primary radiation had extensive intracranial extension. Only one patient underwent post-radiation resection for recurrence.

The problem of intracranial extension in the management of JNA arises in approximately $20 \%$ of cases, based on previous reports [11]. Although theoretically these lesions are surgically resectable by a combined intracranial-extracranial approach, the potential morbidity and mortality may be significantly increased. Jafek et al. [11] reported that radiation therapy may be efficacious in cases of unresected tumor medial to the cavernous sinus. Chandler et al. [6] in a series of 13 surgical cases, stated that radiation therapy offers an attractive alternative in cases with extensive middle fossa or orbital involvement. Ward et al. [20] reported 35 cases of JNA in which surgery was utilized in 33 cases. Twenty percent of these patients had extensive intracranial extension, and one operative death was reported from excessive haemorrhage. The authors state 'It is likewise felt that in the $20 \%$ of cases in which arteriograms show intracranial encroachment, the risks of radiation are less than the possible severe blood loss morbidity, and threat of operative death'.

The three primary complications discussed regarding radiation therapy in adolescents is related to secondary head and neck malignancies, malignant degeneration, and effects on the growth of the craniofacial skeleton. In Cummings series of 55 patients over 26 years, only two malignancies were reported related to radiation therapy, one skin cancer within the radiation field, and one thyroid malignancy. No deleterious effects on craniofacial growth patterns have been reported in the literature regarding radiation therapy for JNA in adolescents. Malignant degeneration in cases of JNA undergoing high-dose radiation therapy have been reported [14]. There is no evidence of short-term complications after radiation therapy in our three patients. Long-term follow-up is essential since secondary malignancies may arise many years following therapy.

In summary, three cases of JNA are presented with extensive intracranial extension. The patients all received primary radiation therapy and had significant resolution of their symptomatology. The tumor mass persisted, although smaller in size by follow-up CT scans. We feel that radiation therapy is a viable alternative to radical surgical excision in selective cases where morbidity and possibly mortality would be increased due to the difficult intracranial location of the tumor. In cases of recurrent disease or persistent tumor which becomes symptomatic, further radiation therapy or surgical resection is a viable alternative. Persistent tumor mass following radiation therapy need not be addressed unless symptoms recur, however, frequent follow-up examinations and repeat CT or MRI scans will be necessary. It is important to manage the JNA patient with a team approach utilizing the skills of the otolaryngologist, neurosurgeon, and radiation oncologist.

\section{References}

1 Andrews, J.C., Fisch, U., Valavanis, A., Aeppli, U. and Makek, M.S. (1989) Surgical management of extensive nasopharyngeal angiofibromas with the infratemporal fossa approach. Laryngoscope, $99,429-437$. 
2 Batsakis, J.G. (1979) Tumors of the Head and Neck, 2nd edn. Williams \& Wilkins Co., Baltimore, pp. 291-312.

3 Benghiat, A. (1986) Juvenile nasopharyngeal angiofibroma treated by radiotherapy. J. Laryngol Otol. 100, 351-356.

4 Briant, T.D.R., Fitzpatrick, P.J. and Berman, J. (1978) Nasopharyngeal angiofibroma: A 20 year study. Laryngoscope 88, 1247-1251.

5 Burkey, B., Koopmann, C.F. and Brunberg, J. (1990) The use of biopsy in the evaluation of pediatric nasopharyngeal masses. Int. J. Pediatr. Otorhinolaryngol. 20, 169-179.

6 Chandler, J.R., Moskowitz, L., Goulding, R. and Quencer, R.M. (1984) Nasopharyngeal angiofibromas: Staging and management. Ann. Otol. Rhinol. Laryngol. 93, 322-329.

7 Close, L.G., Schaefer, S.D., Mickey, B.E. and Manning, S.C. (1989) Surgical management of nasopharyngeal angiofibroma involving the cavernous sinus. Arch. Otolaryngol.-Head Neck Surg. 115, 1091-1095.

8 Cummings, B.J. (1980) Relative risk factors in the treatment of juvenile nasopharyngeal angiofibroma. Head Neck Surg. 3, 21-26.

9 Cummings, B.J. (1983) The treatment of juvenile nasopharyngeal angiofibroma. The case for radiation therapy. J. Laryngol. Otol. (suppl.) 8, 101-102.

10 Cummings, B.J., Blend, R., Fitzpatrick, P., Clark, R., Harwood, A. et al. (1984) Primary radiation therapy for juvenile nasopharyngeal angiofibroma. Laryngoscope 94, 1599-1605.

11 Jafek, B.W., Krekorin, E.A., Kirsch, W.M. and Wood, R.P. (1979) Juvenile nasopharyngeal angiofibroma: The management of intracranial extension. Head Neck Surg. 2, 119-128.

12 Krause, C.J. and Baker, S.R. (1982) Extended transantral approach to pterygomaxillary tumors. Ann. Otol. Rhinol. Laryngol. 91, 395-398.

13 Kremen, A.J. (1953) Surgical management of angiofibroma of the nasopharynx. Ann. Surg. 138, 72.

14 McGahan, R.A., Durrance, F.Y., Parke, R.B., Easley, J.D. and Chou, J.L. (1989) The treatment of advanced juvenile nasopharyngeal angiofibroma. Int. J. Rad. Oncol. Biol. Phys. 17, 1067-1072.

15 Neel, H.B. III, Whicker, J.H., Devine, K.D. and Weiland, L.H. (1973) Juvenile angiofibroma. Review of 120 cases. Am. J. Surg. 126, 547-556.

16 Patterson, C.H. (1965) Juvenile angiofibroma. Arch. Otolaryngol. 76, 167-173.

17 Robinson, A.C.R., Khoury, G.G., Ash, D.V. and Daly, V.D. (1989) Evaluation of response following irradiation of juvenile angiofibromas. Br. J. Radiol. 62, 245-247.

18 Spector, J.G. (1988) Management of juvenile angiofibromata. Laryngoscope 98, 1016-1026.

19 Standefer, J., Holt, G.R., Brown, W.E. Jr. and Gates, G.A. (1983) Combined intracranial and extracranial excision of nasopharyngeal angiofibroma. Laryngoscope 93, 772-779.

20 Ward, P.H., Thompson, R., Calcaterra, T. and Kadin, M.R. (1974) Juvenile angiofibroma: A more rational therapeutic approach based upon clinical and experimental evidence. Laryngoscope 84 , 2181-2194. 